Revista EIA, ISSN 1794-1237 Número 12, p. 125-137. Diciembre 2009

Escuela de Ingeniería de Antioquia, Medellín (Colombia)

\title{
MODELACIÓN DEL COMPORTAMIENTO REOLÓGICO DE ASFALTO CONVENCIONAL Y MODIFICADO CON POLÍMERO RECICLADO, ESTUDIADA DESDE LA RELACIÓN VISCOSIDAD-TEMPERATURA
}

\author{
JALEYDI CÁRDENAS* \\ ELSA FONSECA**
}

\section{RESUMEN}

Las propiedades reológicas del asfalto dependen de las proporciones en que están presentes sus componentes, las cuales varían de acuerdo con el origen de los crudos de petróleo. El comportamiento reológico del ligante tiene una influencia significativa en las propiedades de la mezcla asfalto-agregado (ahuellamiento, fatiga y susceptibilidad térmica). Para describir el comportamiento reológico del asfalto proveniente del Complejo Industrial de Barrancabermeja (CIB) y del asfalto modificado con material reciclado (poliestireno expandido y grano de caucho de llanta reciclada) con control de granulometría y sin él, se realizaron mediciones de viscosidad a diferentes temperaturas (con intervalos de $2^{\circ} \mathrm{C}$ ) con el viscosímetro rotacional Brookfield, y a partir de estos datos se propusieron varias expresiones matemáticas que describen el comportamiento de la viscosidad en términos de la temperatura, se evaluaron en términos del coeficiente de determinación y se hallaron las temperaturas de mezclado y compactación. Los resultados indican que el mejor ajuste en los tres casos corresponde al modelo tipo Arrhenius truncado, con correlaciones entre 0,9945 y 0,9968. Las temperaturas de mezclado y compactación calculadas a partir de este modelo comparadas con las de las normas ASTM D2493 (2001) y AASHTO (2002) resultaron significativamente disímiles para el asfalto modificado (con polímeros de granulometría controlada) pero similares para el asfalto original. De otra parte, con el fin de establecer la estructura química del asfalto original y modificado así como su influencia sobre la viscosidad, se realizó la separación de cada muestra de acuerdo con la norma ASTM D4124 (2001). Los resultados indicaron que sólo ocurrió cambio estructural en el asfalto modificado con polímeros de granulometría controlada, lo que muestra la relación entre la composición química y la viscosidad de los tres ligantes estudiados.

PALABRAS CLAVE: asfalto; asfalto modificado; reología; modelación; estructura química; viscosidad.

* Matemática y Magíster en Ciencias Matemáticas, Universidad Nacional de Colombia. Profesora Departamento de Ciencias Básicas, Universidad de La Salle, Bogotá, Colombia. Grupo de Investigación Indetec. jacardenas@unisalle. edu.co

** Química, Universidad Nacional de Colombia. Magíster en Docencia de la Química, Universidad Pedagógica Nacional. Profesora Departamento de Ciencias Básicas, Universidad de La Salle. Grupo de Investigación Indetec. Bogotá, Colombia.efonsecas@unisalle.edu.co

Artículo recibido 16-VI-2009. Aprobado 14-XII-2009

Discusión abierta hasta junio de 2010 


\section{MODELING OF RHEOLOGICAL BEHAVIOR OF CONVENTIONAL AND RECYCLED POLYMER MODIFIED ASPHALT}

\section{ABSTRACT}

The rheological properties of asphalt are directly related to its chemical composition, which varies with the origin of crude oil. The rheological behavior of the binder has a significant influence on the properties of the asphalt-aggregate mixture (truck-wheel test, fatigue, and thermal susceptibility,). To describe the rheological behavior of asphalt from the Barrancabermeja Industrial Complex (IBC) and the modified asphalt with recycled material (polystyrene and tire rubber) with and without control of particle size, viscosity measurements were conducted at different temperatures (intervals of $2{ }^{\circ} \mathrm{C}$ ) with a Brookfield rotational viscometer, and from these data suggested several mathematical expressions that describe the behavior of viscosity in terms of temperature, were evaluated in terms of the coefficient of determination, and determined the mixing and compaction temperatures. The results indicate that the best fit in the three cases corresponds to the Arrhenius model truncation, correlations between 0.9945 and 0.9968. The mixing and compaction temperatures calculated from this model compared to those of norms ASTM D2493 (2001) and AASHTO (2002) were significantly different for the modified asphalt (polymer-controlled particle size), but similar to the original asphalt. Furthermore, in order to establish the chemical structure of the original and modified asphalt and its influence on the viscosity, the separation was performed for each sample according to ASTM D4124 (2001). The results indicated that only structural changes occurred in the polymer-modified asphalt controlled particle size, which shows the relationship between chemical composition and viscosity of the three binders studied.

KEY WORDS: asphalt; modified asphalt; rheology; modeling; chemical structure; viscosity.

\section{MODELAMENTO DO COMPORTAMENTO REOLÓGICO DE ASFALTO CONVENCIONAL E MODIFICADO COM POLÍMERO RECICLADO}

\section{RESUMO}

As propriedades reológicas do asfalto dependem das proporções em que estão presentes seus componentes, as quais variam de acordo com a origem dos crus de petróleo. O comportamento reológico do ligante tem uma influência significativa nas propriedades da mistura asfalto-agregado (trilha de roda, fadiga e suscetibilidade térmica). Para descrever o comportamento reológico do asfalto proveniente do Complexo Industrial de Barrancabermeja (CIB) e do asfalto modificado com material reciclado (poliestireno expandido e grão de caucho de aro de roda reciclada) com controle de granulometria e sem ele, se realizaram medições de viscosidade a diferentes temperaturas (a intervalos de $2^{\circ} \mathrm{C}$ ) com o viscosímetro rotacional Brookfield, e a partir destes dados se propuseram várias expressões matemáticas que descrevem o comportamento da viscosidade em termos da temperatura, se avaliaram em termos do coeficiente de determinação e se acharam as temperaturas de misturado e compactação. Os resultados indicam que o melhor ajuste nos três casos corresponde ao modelo tipo Arrhenius truncado, correlações entre 0,9945 e 0,9968. As temperaturas de misturado e compactação calculadas a partir deste modelo comparadas com as das normas ASTM D2493 (2001) e AASHTO (2002) resultaram significativamente dissimilares para o asfalto modificado (com polímeros de granulometria controlada) mais similares para o asfalto original. De outra parte, com o fim de estabelecer a estrutura química do asfalto original e modificado assim como sua influência sobre a viscosidade, se realizou a separação de cada amostra de acordo com a norma ASTM D4124. Os resultados indicaram que só ocorreu modificação estrutural no asfalto modificado com polímeros de granulometria controlada, o que amostra a relação entre a composição química e a viscosidade dos três ligantes estudados.

PALAVRAS-CÓDIGO: asfalto; asfalto modificado; reologia; modelação; estrutura química; viscosidade. 


\section{INTRODUCCIÓN}

Los principales problemas que se presentan en los pavimentos asfálticos en servicio son la resistencia a la fatiga y la deformación permanente, como consecuencia de una inadecuada dosificación ligante-agregado, sus interacciones, el método y la temperatura de colocación, mezclado y compactación de la mezcla asfáltica.

Las propiedades deseables en las mezclas asfálticas son: resistentes al desplazamiento, a la fatiga, al deslizamiento, a la deformación plástica, impermeables, durables, grado adecuado de flexibilidad. El desempeño, durabilidad y resistencia de la mezcla asfáltica depende directamente de las propiedades del ligante y el agregado, su interrelación, y de los procesos de elaboración, colocación y compactación (Coinsky et al., 1994).

Los ligantes asfálticos modificados son productos concebidos para superar las propiedades del asfalto original (las cuales dependen de su contenido de asfaltenos, resinas y aceites), mejorando así el desempeño del pavimento a largo plazo. Si bien los modificadores pueden afectar muchas propiedades, la mayoría de ellos intenta reducir la dependencia de la temperatura, el endurecimiento por oxidación del cemento asfáltico y la susceptibilidad a la humedad de la mezcla asfáltica (Coinsky et al., 1994; Sheu, Storm y Mullins, 1995).

El asfalto se clasifica como material viscoelástico porque exhibe características viscosas y elásticas simultáneamente, es decir que es un material de comportamiento intermedio entre el sólido de Hooke (elástico) y el líquido de Newton (viscoso). A temperaturas elevadas $\left(>100^{\circ} \mathrm{C}\right)$, el cemento asfáltico se comporta como un fluido viscoso (muestra la consistencia de un lubricante utilizado como aceite para motores), mientras que a bajas temperaturas $\left(<0{ }^{\circ} \mathrm{C}\right)$ se comporta casi como un sólido elástico (como una banda de goma). Cuando se aplica una carga, el ligante se estira o comprime adoptando diferentes formas. Cuando se retira la carga, retorna a su forma original. A una temperatura intermedia, que es la condición prevista para el pavimento, el cemento asfáltico tiene características de fluido viscoso y sólido elástico (Asphalt Institute, 1995).

Para establecer las temperaturas de mezclado y compactación para el diseño de mezclas asfálticas se busca el empleo de temperaturas equiviscosas, con el fin de minimizar el efecto que puede causar la rigidez del ligante sobre las propiedades volumétricas de la mezcla durante el mezclado en caliente en planta, independiente del tipo de ligante, original o modificado y de la gradación del agregado (Asphalt Institute, 1995).

El viscosímetro rotacional (RTV) consiste en un cilindro rotacional coaxial que mide la viscosidad por medio de un torque requerido para rotar un eje; sumergido en la muestra de asfalto, permite determinar la curva reológica del asfalto. Para caracterizar las propiedades viscoelásticas de los bitúmenes se recomienda el uso del reómetro de corte dinámico DSR, el cual mide la respuesta de la deformación específica de corte del espécimen sometido a un torque, y finalmente el reómetro de viga flexible (BBR) permite caracterizar las propiedades elásticas del bitumen a bajas temperaturas.

El Instituto del Asfalto recomendó en 1962 emplear la viscosidad Saybolt Furol del asfalto como criterio fundamental para determinar las temperaturas de mezclado y compactación de la mezcla las correspondientes a un rango de viscosidad de $170 \pm 20$ centistokes y como temperatura de compactación la temperatura de mezclado y de $280 \pm 30$ centistokes. Estos valores siguieron vigentes incluso durante el furor de la década de los noventa de las especificaciones Superpave para el diseño de mezclas asfálticas, en las cuales se mantuvieron los valores establecidos por el Instituto del Asfalto en los sesenta, pero empleando para su determinación al viscosímetro rotacional RTV y cambiando las unidades de viscosidad en centistokes por unidades del Sistema Internacional: $(0,17 \pm 0,02)$ Pa.s para la temperatura de mezclado y $(0,28 \pm 0,03)$ Pa.s para la 
temperatura de compactación. Estos valores han sido de uso generalizado desde entonces (Reinke, 2000) y hacen parte de las especificaciones de la norma ASTM D 2493 "Standard viscosity-temperature chart for asphalts", incluso aparecen en la actualización de 2006 del Asphalt Institute.

Yildirim, Ideker y Haztlett (2006) realizaron un estudio con 50 ligantes asfálticos modificados y sin modificar disponibles comercialmente, para verificar la validez de la determinación de las temperaturas de mezclado y compactación por medio de las curvas reológicas de acuerdo con las directrices de la norma de la American Society for Testing and Materials ASTM D 2493. Los autores utilizaron la ley de potencia para establecer una relación lineal entre los valores de viscosidad (log-log) y los de temperatura (log), concluyendo que los valores de temperatura de mezclado y compactación obtenidos por métodos convencionales para los asfaltos modificados son más altos de lo requerido en la vía.

Otro trabajo sobre las diferencias entre las temperaturas de mezclado y compactación de asfaltos modificados y originales fue el de Delmar Salomón Coe y Huachun Zhai Cui (2004), quienes emplearon la viscosimetría rotacional para determinar la energía de activación de flujo de ligantes asfálticos modificados y sin modificación, provenientes de diferentes crudos. A partir de la ecuación de Arrhenius, establecieron que la energía de activación de flujo para todos los ligantes asfálticos se encuentra entre $44 \mathrm{~kJ} / \mathrm{mol}$ y $90 \mathrm{~kJ} / \mathrm{mol}$, estableciendo que a partir de fuentes de asfaltos y tipos de polímeros diferentes resultan energías de activación de flujo distintas. Una explicación posible a este hecho tiene que ver con la diversidad presente en la composición de los ligantes asfálticos y con la interacción entre los componentes de polímeros y asfaltos. Además, encontraron que la energía de activación de flujo disminuye cuando el contenido polimérico rebasa la concentración de polímero crítica, lo que se debe en principio a la variación de interacciones entre las moléculas de los polímeros y los componentes de los asfaltos, y aumenta debido al envejecimiento por oxidación, ya que a partir de la oxidación se incrementa el número de moléculas polares en los ligantes asfálticos, lo que resulta en un incremento de las fuerzas intermoleculares.

La composición química (estructura y asociaciones moleculares) del ligante asfáltico influye en las propiedades físicas y mecánicas de las mezclas asfálticas, así como en su desempeño, por lo que su estudio reviste gran importancia. Así mismo, las propiedades reológicas del asfalto dependen de las proporciones en que están presentes sus componentes, las cuales varían de acuerdo con el origen de los crudos de petróleo (Hoiberg, 1965).

Históricamente se han estudiado dos modelos principales de estructura química del asfalto: el modelo micelar de Nellensteyn y el modelo continuo SHRP (Wahr, 2002). En el modelo micelar, se supone que el asfalto está formado por grandes moléculas de asfaltenos rodeadas por aromáticos polares y no polares, suspendidas en aceites saturados, todos los constituyentes peptizados en una solución coloidal. Los saturados y aromáticos se pueden considerar como los medios de dispersión de los aromáticos polares, que son los responsables del comportamiento viscoelástico del asfalto a temperatura ambiente (Montejo, 2002).

Los modelos de SHRP no están de acuerdo con el modelo micelar de "supermolécula"; por el contrario, se considera al asfalto simplemente como un compuesto de dos fases: una polar y una no polar. Sus propiedades dependen de la compatibilidad y habilidad de los constituyentes para coexistir sin transformación de fase en el tiempo, también están influenciadas por el grado de aromaticidad. La estructura química primaria de los cementos asfálticos incluye: anillos aromáticos, anillos cíclicos (aromáticos nafténicos), alifáticos (alcanos), heteroátomos $(\mathrm{O}, \mathrm{N}, \mathrm{S})$ que forman asociación entre moléculas (inducen polaridad) e influyen en forma significativa en el comportamiento mecánico del asfalto, y metales (Va, Ni, Fe) que tienen influencia sobre 
el envejecimiento y la huella dactilar del asfalto (Wahr, 2002).

En este trabajo, se proponen varias expresiones que modelan matemáticamente la viscosidad del asfalto original y modificado en términos de la temperatura y se evalúa el ajuste de estos modelos a los datos experimentales obtenidos con el viscosímetro rotacional Brookfield entre $80{ }^{\circ} \mathrm{C}$ y $200{ }^{\circ} \mathrm{C}$ a intervalos de $2{ }^{\circ} \mathrm{C}$, definiendo a partir del modelo de mejor comportamiento las temperaturas de mezclado y compactación de los ligantes. Así mismo, se determina la relación entre la curva reológica y la composición química de los asfaltos estudiados.

\section{MATERIALES Y MÉTODOS}

El asfalto CIB empleado para el estudio proviene del complejo industrial de Barrancabermeja (Santander, Colombia); se clasifica por su penetración de acuerdo con la norma vigente del Instituto Nacional de Vías INVIAS como asfalto 70-90. Los polímeros empleados como modificadores fueron poliestireno expandido y llanta de caucho reciclados.

\subsection{Modificación del asfalto}

El asfalto CIB fue modificado empleando el dispersor de asfaltos del Laboratorio de Pavimentos de la Universidad de La Salle (Bogotá). La primera modificación se realizó con $14 \%$ de llanta y 1\% de poliestireno molidos antes de ser incorporados al asfalto, mientras que en la segunda se optimizó la granulometría de los polímeros, de tal forma que el tamaño del poliestireno expandido estuviera entre el tamiz No. 10 y el tamiz No. 40 (Figueroa y Reyes, 2005), mientras que la llanta fuera de tamaño inferior a $40 \mu \mathrm{m}$ malla 40 (Ocampo, Caicedo y González, 2002a, 2002b). El tamaño de partícula seleccionado para cada modificador permite la formación de una red polimérica homogénea entre el asfalto y el polímero, que no se obtiene sin este control granu- lométrico, como se ha verificado por microscopia electrónica (Figueroa y Fonseca, 2008; Figueroa, Fonseca y Reyes, 2009).

\subsection{Determinación de curvas reológicas}

La reología del asfalto convencional y modificado fue evaluada con el viscosímetro Brookfield DV III del Laboratorio de Pavimentos de la Universidad de La Salle (Bogotá), realizando inicialmente un barrido general y luego lecturas de viscosidad cada $2{ }^{\circ} \mathrm{C}$ entre $80^{\circ} \mathrm{C}$ y $200{ }^{\circ} \mathrm{C}$.

\subsection{Determinación de la composición química}

La composición química de los ligantes fue establecida por medio del fraccionamiento en saturados, aromáticos, resinas y asfaltenos (método SARA). El ensayo, realizado en los laboratorios de Química de la Universidad de La Salle de acuerdo con las especificaciones de la norma ASTM D-4124, consiste en la separación del asfalto en asfaltenos y maltenos por precipitación de aquellos con n-heptano, n-hexano o n-pentano. Los maltenos se fraccionan después a través de una columna de alúmina con solventes de polaridad creciente.

\section{RESULTADOS Y DISCUSIÓN}

A continuación se presentan los resultados obtenidos para cada uno de los ligantes estudiados.

\subsection{Curvas reológicas}

En las figuras 1 a 3 se presenta la curva reológica obtenida para el asfalto CIB original, modificado sin granulometría controlada. Se puede observar que para las tres muestras de ligante el comportamiento reológico representado por la gráfica directa de viscosidad contra tenmperatura es muy similar. 


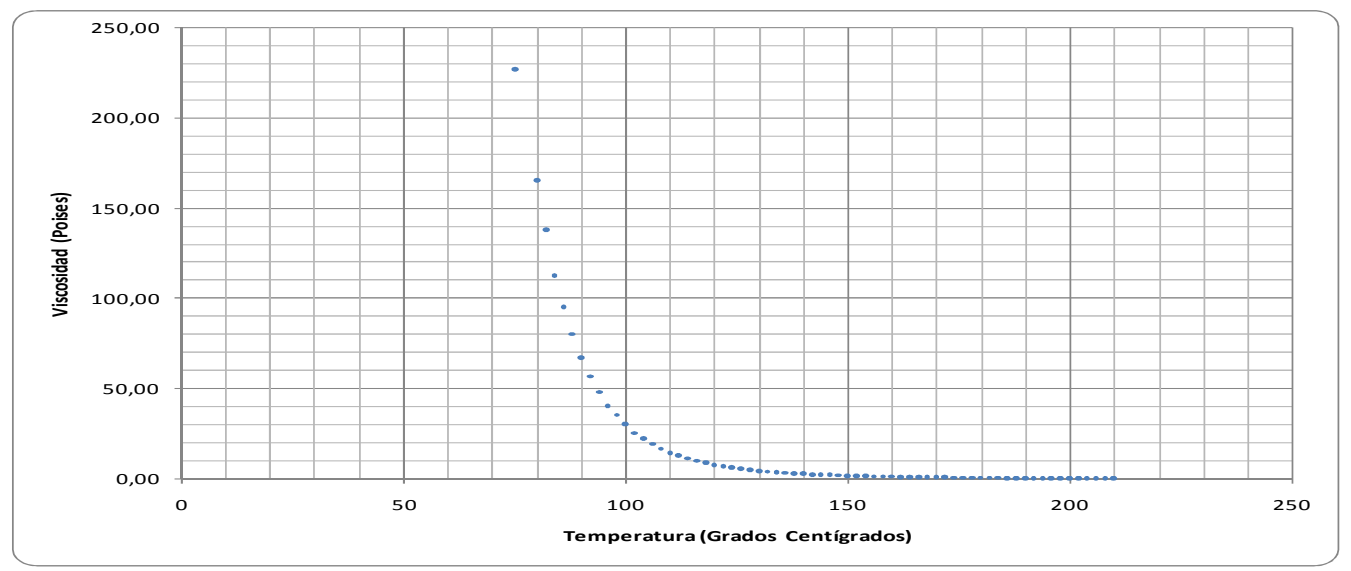

Figura 1. Curva reológica del asfalto CIB original

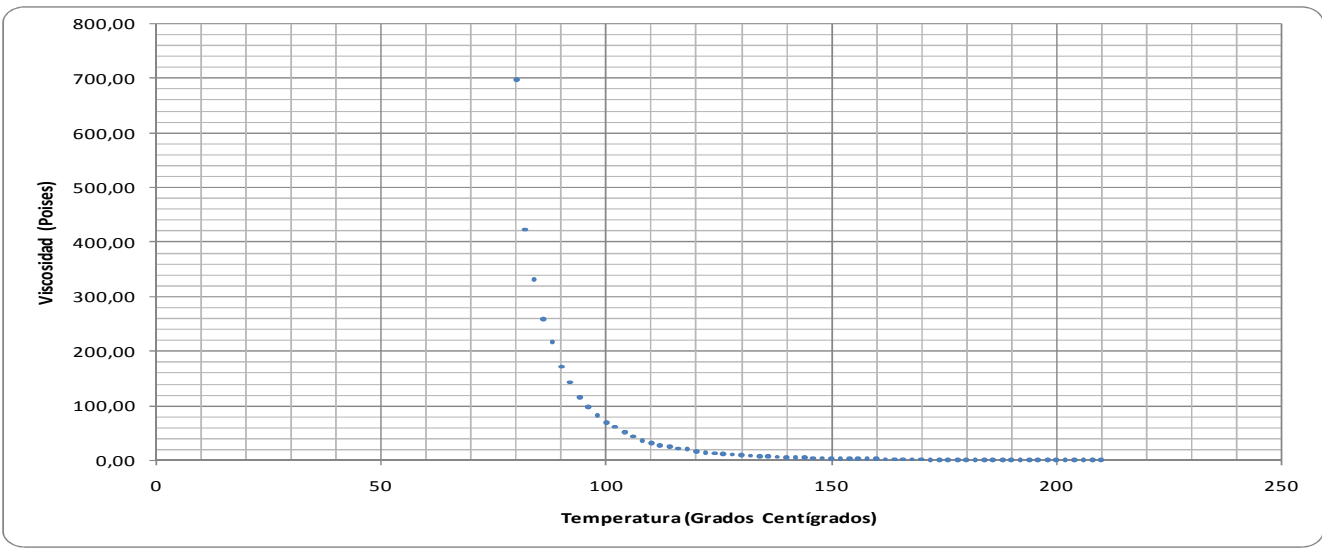

Figura 2. Curva reológica del asfalto CIB modificado sin granulometría controlada

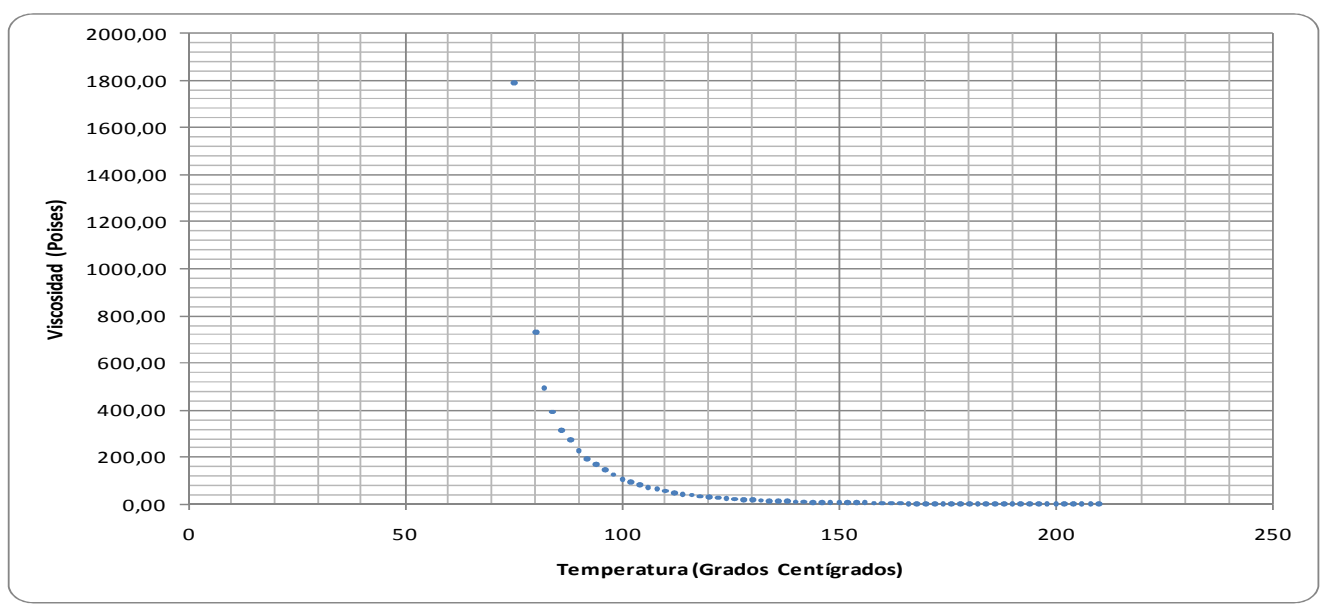

Figura 3. Curva reológica del asfalto CIB modificado con granulometría controlada 
A partir de los datos observados en el laboratorio, se propusieron varias expresiones que modelan matemáticamente la viscosidad en términos de la temperatura y se evaluó el ajuste de estos modelos. Se procedió por interpolación polinómica, luego haciendo estimaciones con funciones exponenciales y posteriormente estimaciones a partir de ecuaciones empíricas propias de los estudios reológicos, considerando además sus correspondientes versiones truncadas para disminuir el efecto de las viscosidades altas obtenidas a temperaturas inferiores a $130{ }^{\circ} \mathrm{C}$

\subsection{Interpolación polinómica}

El primer ajuste se hizo mediante interpolación con los datos de barrido de la curva reológica a $80,100,120,140,160,180$ y 200 grados Celsius, obteniendo una expresión polinómica de grado 6 para la viscosidad (poises) en términos de la temperatura para cada asfalto. Este tipo de ajuste mostró ser deficiente tanto para el asfalto original como para los modificados, ya sea porque la viscosidad calculada mediante el polinomio propuesto resulta negativa en ciertos valores de la temperatura o porque el valor obtenido de la viscosidad no concuerda con los datos experimentales.

Para mejorar esta clase de deficiencias es necesario considerar más datos intermedios en la construcción del polinomio, pero esto conlleva un aumento proporcional en su grado y hace demasiado engorroso el manejo práctico de la expresión final que relaciona y modela la viscosidad en términos de la temperatura.

\subsection{Relaciones exponenciales y empiricas}

De acuerdo con los datos observados y pensando en una expresión de manejo práctico, se plantearon tres modelos generales de tipo exponencial y se estimaron los parámetros usando regresión por mínimos cuadrados.
Se comenzó con una expresión exponencial lineal, donde el exponente es una función lineal de la temperatura:

$$
\eta(T)=e^{a+b T}
$$

Sin embargo, la estimación de los parámetros se ve sensiblemente afectada, ya que las viscosidades observadas a temperaturas inferiores a $130{ }^{\circ} \mathrm{C}$ son grandes en comparación con los demás datos. Esto llevó a plantear una versión truncada del modelo, donde sólo se consideraron las viscosidades observadas a temperaturas entre $130^{\circ} \mathrm{C}$ y $210^{\circ} \mathrm{C}$ (rango donde por lo general se encuentran las temperaturas de mezclado y compactación).

Con el fin de determinar los efectos que tiene incrementar el grado del polinomio en el exponente, se propuso un modelo exponencial cuadrático y su correspondiente versión truncada, pues la estimación de parámetros nuevamente se ve afectada por los valores iniciales.

$$
\eta(T)=e^{a+b T+c T^{2}}
$$

Dado que en general el incremento en el grado del exponente no mejora el ajuste, se procedió a disminuir el grado y a considerar un modelo exponencial de la forma:

$$
\eta(T)=e^{1 / T}
$$

o más específicamente, un modelo con la forma de la ecuación empírica de Arrhenius, estimando en forma directa los parámetros allí involucrados:

$$
\eta(T)=A e^{E / R T}
$$

y se consideró además su versión truncada con el fin de hacer óptimo el ajuste.

Por último, a partir de la ley de potencia, citada en la norma ASTM D 2493 (2006) (Yildirim, Ideker y Haztlett, 2006; Lefebvre y Robertson, 1970), se propone un modelo acorde con esta relación junto con su respectivo truncamiento:

$$
\eta(T)=A T^{\eta}
$$


Tabla 1. Comparación entre modelos en términos de sus coeficientes de determinación

\begin{tabular}{|l|c|c|c|}
\cline { 2 - 4 } \multicolumn{1}{c|}{} & Asfalto 1 & Asfalto 2 & Asfalto 3 \\
\hline Modelo exponencial lineal & 0,9610 & 0,9536 & 0,9568 \\
\hline Modelo exponencial lineal truncado & 0,9917 & 0,9873 & 0,9839 \\
\hline Modelo exponencial cuadrático & 0,9437 & 0,9356 & 0,9392 \\
\hline Modelo exponencial cuadrático truncado & 0,9875 & 0,9815 & 0,9781 \\
\hline Modelo tipo Arrhenius & 0,9866 & 0,9813 & 0,9832 \\
\hline Modelo tipo Arrhenius truncado & 0,9968 & 0,9957 & 0,9945 \\
\hline Modelo tipo ley de potencia & 0,9754 & 0,9689 & 0,9716 \\
\hline Modelo tipo ley de potencia truncado & 0,9948 & 0,9921 & 0,9907 \\
\hline
\end{tabular}

\subsection{Comparación entre modelos}

En términos de sus coeficientes de determinación, los modelos que mostraron mejores ajustes con los datos observados para los tres tipos de asfaltos estudiados fueron en su orden: el de Arrhenius, el de la ley de la potencia y el exponencial lineal, todos en sus versiones truncadas, como indica la tabla 1.

\subsection{Resultados comparados}

Se presentan la función de ajuste del modelo de Arrhenius truncado, el coeficiente de determinación, el error típico, la energía de activación calcula- da y la comparación entre los datos observados y los estimados, para cada uno de los asfaltos evaluados (ecuaciones 6 a 15). Las figuras 4 a 6 muestran los casos de comparación entre datos experimentales y los estimados según Arrhenius truncado.

Para el asfalto original se obtuvo:

$\eta(T)=3,7269 \times 10^{-7} e^{54400,9482 / 8.31434} \mathrm{~T}$

$R^{2}=0,9968 ;$ Error típico: 0,0458

$\mathrm{E}_{a=54400,9482}$

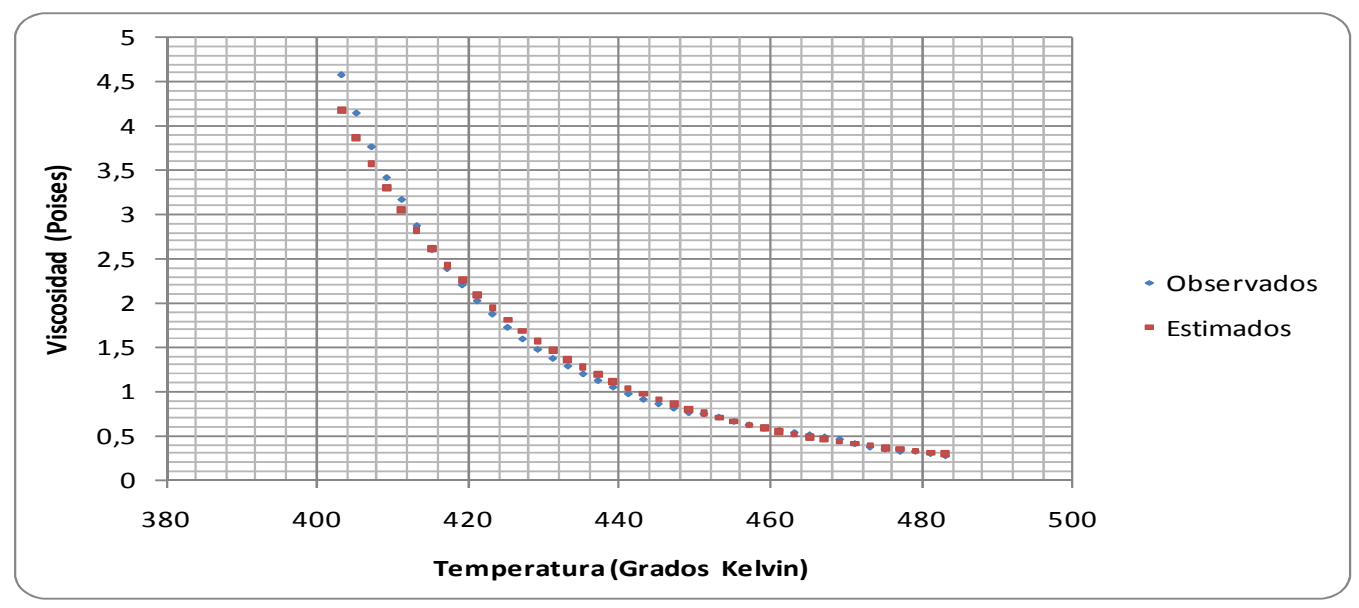

Figura 4. Comparación de datos experimentales con estimados a partir del modelo Arrhenius truncado. Asfalto original y asfalto modificado sin granulometría controlada 


$$
\begin{aligned}
& \eta(T)=3,3729 \times 10^{-7} e^{57154,8978 / 8.31434 \mathrm{~T}} \\
& R^{2}=0,9957 ; \text { Error típico: } 0,056 \\
& E_{a}=57154,8978
\end{aligned}
$$$$
\eta(T)=1,02077 \times 10^{-6} e^{55509,3221} / 8.31434 \mathrm{~T}
$$$$
\text { (10) } \quad R^{2}=0,9945 \text {; Error típico: 0,0619 }
$$$$
\text { (11) } \mathrm{E}_{a=55509,3221}
$$

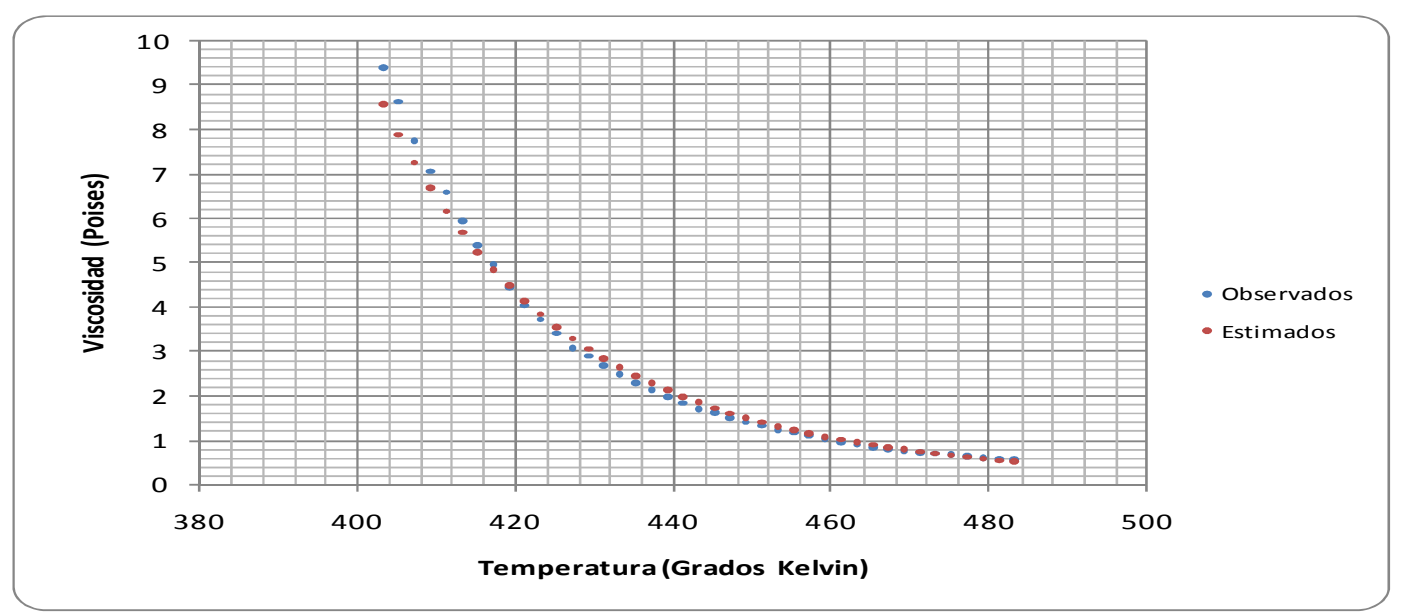

Figura 5. Comparación de datos experimentales con estimados a partir del modelo Arrhenius truncado. Asfalto modificado sin granulometría controlada y asfalto modificado con granulometría

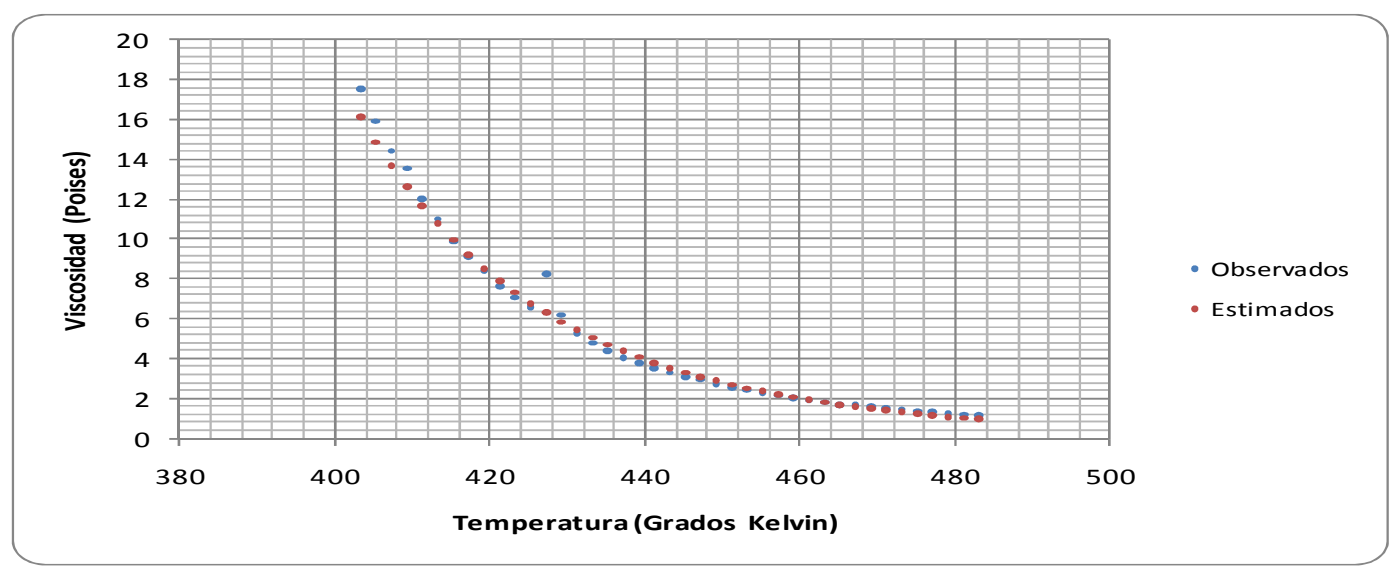

Figura 6. Comparación de datos experimentales con estimados a partir del modelo Arrhenius truncado. Asfalto modificado con granulometría controlada

Para el asfalto CIB convencional la energía de activación de flujo calculada fue de 54,4 KJ/mol, para el asfalto CIB modificado con polímeros sin granulometría controlada fue de $57,1 \mathrm{KJ} / \mathrm{mol}$, mientras que para el asfalto CIB modificado con polímeros con granulometría controlada fue de $55,5 \mathrm{KJ} / \mathrm{mol}$. El resultado anterior indica que los polímeros añadidos (poliestireno expandido y llanta) sin granulometría controlada que corresponden a un $15 \%$ en peso del total del ligante aumentan la energía de activación 
de flujo, mientras que la energía de activación de flujo del asfalto modificado con polímeros de granulometría controlada resultó del mismo orden que la del asfalto original.

Los resultados de energía de activación de flujo indican que el asfalto CIB modificado tiene una sensibilidad a la temperatura similar a la del asfalto CIB convencional. Según Delmar Salomón Coe y Huachun Zhai Cui (2004), una energía de activación baja indica que el ligante tiene menor sensibilidad a la temperatura que un ligante con energía de activación alta.

\subsection{Determinación de temperaturas de mezclado y compactación}

Con la expresión matemática correspondiente al modelo Arrhenius truncado se calculó el intervalo o rango de temperaturas de mezclado y compactación. Además se compararon estos valores con los obtenidos a partir del método usual (norma ASTM D 2493) que considera únicamente de 4 a 6 puntos en la curva reológica.

Tabla 2. Temperaturas de mezclado y compactación para el asfalto CIB original, determinadas con la norma ASTM D 2493

y con el método de Arrhenius truncado

\begin{tabular}{|l|c|c|}
\hline \multicolumn{3}{|c|}{ Rango de temperaturas de mezclado } \\
\hline Viscosidad & $\begin{array}{c}\text { Métododo usual } \\
\left({ }^{\circ} \mathbf{~ C )}\right.\end{array}$ & $\begin{array}{c}\text { Método AT } \\
\left({ }^{\circ} \mathbf{~ C )}\right.\end{array}$ \\
\hline $1,5 \mathrm{P}$ & 156,29 & 157,08 \\
\hline $1,9 \mathrm{P}$ & 150,29 & 150,49 \\
\hline
\end{tabular}

\begin{tabular}{|l|c|c|}
\hline \multicolumn{3}{|c|}{ Rango de temperaturas de compactación } \\
\hline Viscosidad & $\begin{array}{c}\text { Métododo usual } \\
\mathbf{(}^{\circ} \mathbf{~ C )}\end{array}$ & $\begin{array}{c}\text { Método AT } \\
\left({ }^{\circ} \mathbf{~ C )}\right.\end{array}$ \\
\hline 2,5 P & 143,32 & 143,09 \\
\hline 3,1 P & 137,86 & 137,47 \\
\hline
\end{tabular}

Para el asfalto CIB original, las temperaturas de mezclado y compactación calculadas a partir del modelo propuesto son similares a las obtenidas mediante el método tradicional (tabla 2).
Tabla 3. Temperaturas de mezclado y compactación para el asfalto CIB modificado con polímeros sin granulometría controlada, determinadas con la norma ASTM D 2493

y con el método de Arrhenius truncado

\begin{tabular}{|l|c|c|}
\hline \multicolumn{3}{|c|}{ Rango temperaturas de mezclado } \\
\hline Viscosidad & $\begin{array}{c}\text { Método } \\
\text { usual } \\
\left({ }^{\circ} \mathbf{C}\right)\end{array}$ & $\begin{array}{c}\text { Método } \\
\mathbf{A T} \\
\left({ }^{\circ} \mathbf{~ C )}\right.\end{array}$ \\
\hline $1,5 \mathrm{P}$ & 172,22 & 175,91 \\
\hline $1,9 \mathrm{P}$ & 166,63 & 169,08 \\
\hline
\end{tabular}

\begin{tabular}{|l|c|c|}
\hline \multicolumn{3}{|c|}{ Rango temperaturas de compactación } \\
\hline Viscosidad & $\begin{array}{c}\text { Método } \\
\text { usual } \\
\left({ }^{\circ} \mathrm{C}\right)\end{array}$ & $\begin{array}{c}\text { Método } \\
\text { AT } \\
\left.\mathbf{(}^{\circ} \mathrm{C}\right)\end{array}$ \\
\hline $2,5 \mathrm{P}$ & 160,13 & 161,41 \\
\hline $3,1 \mathrm{P}$ & 155,04 & 155,58 \\
\hline
\end{tabular}

Para el asfalto CIB modificado con polímeros sin granulometría controlada, las temperaturas de mezclado y compactación calculadas a partir del modelo propuesto también son similares a las obtenidas mediante el método tradicional (tabla 3).

Tabla 4. Temperaturas de mezclado y compactación para el asfalto CIB modificado con polímeros de granulometría controlada, determinadas con la norma ASTM D 2493 y por el método de Arrhenius truncado

\begin{tabular}{|l|c|c|}
\hline \multicolumn{3}{|c|}{ Rango temperaturas de mezclado } \\
\hline Viscosidad & $\begin{array}{c}\text { Método } \\
\text { usual } \\
\mathbf{}^{\circ} \mathbf{~ C )}\end{array}$ & $\begin{array}{c}\text { Método } \\
\left({ }^{\circ} \mathbf{~ C )}\right.\end{array}$ \\
\hline $1,5 \mathrm{P}$ & 189,02 & 196,99 \\
\hline $1,9 \mathrm{P}$ & 183,29 & 189,29 \\
\hline
\end{tabular}

\begin{tabular}{|l|c|c|}
\hline \multicolumn{3}{|c|}{ Rango temperaturas de compactación } \\
\hline Viscosidad & $\begin{array}{c}\text { Método } \\
\text { Musual } \\
\left({ }^{\circ} \mathrm{C}\right)\end{array}$ & $\begin{array}{c}\text { Método } \\
\left({ }^{\circ} \mathrm{C}\right)\end{array}$ \\
\hline $2,5 \mathrm{P}$ & 176,63 & 180,66 \\
\hline $3,1 \mathrm{P}$ & 171,42 & 174,42 \\
\hline
\end{tabular}


Para el asfalto CIB modificado con polímeros con granulometría controlada, las temperaturas de mezclado y compactación calculadas a partir del modelo propuesto difieren de las obtenidas por el método tradicional en un rango de entre $4{ }^{\circ} \mathrm{C}$ y $9^{\circ} \mathrm{C}$ (tabla 4$)$.

El comportamiento anterior corresponde a lo observado por microscopia electrónica de barrido SEM, donde se comprobó que la morfología del asfalto CIB original y la del modificado con granulometría polimérica controlada es homogénea y conserva sus propiedades en el tiempo, mientras que el asfalto modificado sin granulometría controlada no presenta una estructura homogénea, por lo que con el tiempo o con el cambio de la temperatura se separa en dos fases: fase polímero modificador y fase ligante (Figueroa y Fonseca, 2008), predominando las propiedades del asfalto original.

\subsection{Determinación de la composición química del asfalto CIB original y CIB modificado}

A continuación se presentan los resultados obtenidos en el fraccionamiento por el método SARA únicamente para el asfalto CIB original y para el modificado con polímeros de granulometría controlada, ya que el asfalto modificado sin granulometría controlada presentó un análisis SARA muy similar al del asfalto original.

Tabla 5. Resultados del análisis SARA realizado al asfalto CIB convencional y modificado

\begin{tabular}{|l|c|c|c|c|c|c|}
\hline \multicolumn{1}{|c|}{ Asfalto } & Saturados & Aromáticos & Resinas & Asfaltenos & Maltenos & IC \\
\hline CIB convencional & 25,16 & 37,62 & 28,83 & 8,39 & 91,61 & 0,50 \\
\hline CIB modificado & 13,75 & 20,98 & 47,43 & 17,84 & 82,16 & 0,46 \\
\hline
\end{tabular}

Los resultados anteriores muestran que el asfalto modificado presenta una mayor cantidad de resinas y un menor contenido de saturados y aromáticos que el asfalto original, y dado que en el modelo micelar del asfalto (Hoiberg, 1965) los saturados y aromáticos son los medios de dispersión de las resinas (aromáticos polares), se espera que el asfalto modificado presente temperaturas equiviscosas más elevadas que el asfalto original y en consecuencia, mayores temperaturas de mezclado y compactación.

El índice de inestabilidad coloidal de la tabla 5, se calculó a partir de la ecuación 15:

IC $=\frac{\text { Constituyentes floculados }}{\text { Constituyentes dispersados }}=\frac{\text { Saturados }+ \text { Asfaltenos }}{\text { Aromáticos }+ \text { Resinas }}$
De acuerdo con los índices de inestabilidad coloidal (IC) calculados, tanto el asfalto original como el modificado se clasifican como asfaltos tipo sol-gel, ideales para ser usados en pavimentación por tener una proporción adecuada de maltenos y asfaltenos, siendo más equilibrada la del asfalto modificado. El asfalto CIB modificado es más rígido que el CIB convencional puesto que tiene un mayor porcentaje de asfaltenos y menos saturados que el CIB convencional.

En la tabla 6 se presentan las proporciones entre las diferentes fracciones obtenidas por el método SARA para el asfalto CIB convencional y el modificado con granulometría controlada. 
Tabla 6. Razones entre las fracciones obtenidas por análisis SARA

\begin{tabular}{|c|c|c|c|c|c|}
\hline \multicolumn{1}{|c|}{ Asfalto } & $\frac{\text { Saturados }}{\text { Resinas }}$ & $\frac{\text { Saturados }}{\text { Aromáticos }}$ & $\frac{\text { Aromáticos }}{\text { Resinas }}$ & $\frac{\text { Resinas }}{\text { Aromáticos }}$ & $\frac{\text { Resinas }}{\text { Asfaltenos }}$ \\
\hline CIB convencional & 0,87 & 0,67 & 1,30 & 0,77 & 3,43 \\
\hline CIB modificado & 0,29 & 0,66 & 0,44 & 2,26 & 2,66 \\
\hline
\end{tabular}

Los resultados obtenidos para el asfalto modificado (tablas 5 y 6) corroboran el postulado de Simpson y Griffin en el que se relaciona la viscosidad con la composición química del asfalto: si la razón saturados/aromáticos permanece constante y aumenta el porcentaje de resinas entonces la viscosidad aumentará (Ver curva reológica).

\section{CONCLUSIONES}

Para los tres asfaltos estudiados el modelo que presentó un mejor ajuste a los datos experimentales observados de viscosidad en términos de la temperatura fue del tipo Arrhenius truncado $\left(R^{2}\right.$ entre 0,9945 y 0,9968; errores típicos entre 0,0458 y 0,0619).

Las energías de activación de flujo calculadas con el modelo propuesto resultaron similares para los tres asfaltos estudiados ( $E_{a}$ entre 54 y $\left.57 \frac{\mathrm{kJ}}{\mathrm{mol} \mathrm{K}}\right)$.

El asfalto CIB original y el modificado con polímeros de granulometría controlada presentaron un aspecto homogéneo monofásico, con un aumento significativo en los valores de viscosidad del modificando frente al original; mientras que el asfalto modificado con polímeros sin granulometría controlada presentó un aspecto heterogéneo multifásico y valores de viscosidad similares a los del asfalto original. Lo anterior indica que la morfología de los tres ligantes estudiados está relacionada con las temperaturas de mezclado y compactación calculadas a partir del modelo propuesto.

La composición química de los asfaltos evaluados a través del análisis SARA indicó que únicamente ocurrió modificación estructural en el asfalto modificado con polímeros de granulometría controlada, lo que muestra la relación entre la composición química y la viscosidad de los tres ligantes estudiados.

Las temperaturas de mezclado y compactación calculadas a partir del modelo propuesto comparadas con las de la norma ASTM D2493 (2001) resultaron significativamente disímiles para el asfalto modificado (con polímeros de granulometría controlada) pero similares para el asfalto original, lo que corrobora lo anotado por algunos autores como Olazábal et al. (2005), Yildirim, Ideker y Haztlett (2006) con respecto a que para asfaltos modificados se debe buscar un método más eficiente que el de la curva reológica de 4-6 puntos para determinar las temperaturas de mezclado y compactación.

\section{BIBLIOGRAFÍA}

American Association of State Highway and Transportation Officials (AASHTO). "Standard practice for mixture conditioning of hot mix asphalt". AASHTO R-30 2002.

Asphalt Institute. Asphalt Institute Technical Bulletin: "Laboratory mixing and compaction temperatures". Lexington, Kentucky USA, 2006. 5 p.

Superpave performance, graded asphalt binder specification and testing. Series No 1 (SP-1) Lexington, Kentucky USA, 1995. pp. 6-10, 20-35.

ASTM International Standards worldwide. "Standard viscosity-temperature chart for asphalts”. ASTM D2493 (2001).

. "Standard test methods for separation of asphalt into four fractions”. ASTM D4124 (2001).

Coe, D. S. and Cui, Z. H. (2003). "Determinación de la energía de activación de flujo en ligantes asfálticos”. 
XII Congreso Ibero-Latinoamericano del Asfalto CILA, Quito (Ecuador), Disponible en www.technopave.com/ publications/CILA-2003

Coinsky, R.; Huber, G.; Anderson, M.; Kennedy, T.; Solaiman, M. The Superpave mix design manual for new construction. Strategic Highway Research Program SHRP-A-407, 1994. chapters 1 and 2.

Figueroa A. S. y Fonseca E. B. (2008). Comportamiento mecánico de una mezcla asfáltica MDC-2 modificada con poliestireno y polvo de llanta. Facultad de Ingeniería Civil, Departamento de Ciencias Básicas, Universidad de La Salle. (Informe técnico de proyecto). Bogotá.

Figueroa A. S.; Fonseca E. B. y Reyes F. A. (2009). "Caracterización fisicoquímica y morfológica de asfaltos modificados con material reciclado". Ingeniería y Universidad, vol. 13, pp. 50-66.

Figueroa, A. S. y Reyes, F. A.. Asfaltos modificados con poliestireno. Bogotá: Universidad de La Salle, Pontificia Universidad Javeriana, 2005. 83 p.

Hoiberg, A. Bituminous materials. Vol. 2. Asfaltos. New York: John Wiley \& Sons, 1965.

Montejo, A. Ingeniería de pavimentos: evaluación estructural, obras de mejoramiento y nuevas tecnologías, Tomo 2, $3^{\text {a }}$ ed. Bogotá: Universidad Católica de Colombia, 2006, pp. 91-93.
Ocampo, M.; Caicedo, B. y González, D. (2002a) Experiencia colombiana sobre el empleo de grano de caucho reciclado proveniente de llantas usadas. Ponencia presentada en Terceras Jornadas Internacionales del Asfalto. Agosto 21, 22, 23 de 2002, Universidad del Cauca, Popayán.

(2002b). Mezclas asfálticas mejoradas con caucho molido proveniente de llantas. Revista de Ingeniería. 2002, núm. 16: 175-80.

Olazábal P.; Larsen D.; Cortizo M. S. y Alessandrini J. L. "Análisis del efecto térmico en la reología de asfaltos modificados". Universidad Nacional de La Plata, 2005.

Reinke G. (2000). "Prepared discussion to mixing and compaction temperatures for superpave mixes". Asphalt Paving Technology, vol. 70, pp. 368-402.

Sheu E. Y.; Storm D. A. and Mullins O. C. "Asphaltenes fundamentals and applications", New York: Plenum Press, 1995. pp. 1-4.

Wahr, C. Materiales bituminosos 2002 (en línea). Disponible en www. e-asfalto.com.

Yildirim Y.; Ideker J. and Haztlett D. (2006). Evaluation of viscosity values for mixing and compactation temperatures". Journal of Materials in Civil Engineering, vol. 18, No. 4 (July-August) pp. 545-553. 